\title{
Multiclass Brain Glioma Tumor Classification Using Block-Based 3D Wavelet Features of MR Images
}

\author{
Ghazanfar Latif ${ }^{1,3^{*}}$, M. Mohsin Butt ${ }^{2}$, Adil H. Khan ${ }^{3}$, Omair Butt ${ }^{3}$, D. N. F. Awang Iskandar ${ }^{1}$ \\ ${ }^{1}$ Faculty of Computer Science and Information Technology, Universiti Malaysia Sarawak, Malaysia \\ ${ }^{2}$ College of Applied and Supporting Studies, King Fahd University of Petroleum and Minerals, Dhahran, KSA \\ ${ }^{3}$ College of Electrical Engineering, Prince Mohammad bin Fahd University, Al Khobar, Saudi Arabia \\ e-mail: ghazanfar.latif@nu.edu.pk
}

\begin{abstract}
With the advent of more powerful computing devices, system automation plays a pivotal role. In the medical industry, automated image classification and segmentation is an important task for decision making about a particular disease. In this research, a new technique is presented for classification and segmentation of low-grade and high-grade glioma tumors in Multimodal Magnetic Resonance (MR) images. In the proposed system, each multimodal MR image is divided into small blocks and features of each block are extracted using three Dimensional Discrete Wavelet Transform (3D DWT). Random Forest classifier is used for the classification of multiple Glioma tumor classes, then segmentation is performed by reconstructing the MR image based on the classified blocks. MICCAI BraTS dataset is used for testing the proposed technique and experiments are performed for Low Grade Glioma (LGG) and High Grade Glioma (HGG) datasets. The results are compared with different classifiers e.g. multilayer perceptron, radial basis function, Naïve Bayes, etc., After careful analysis, Random Forest classifier provided better precision by securing average accuracy of $89.75 \%$ and $86.87 \%$ is obtained for HGG and LGG respectively.
\end{abstract}

Keywords-brain tumor classification; glioma; multimodal brain MRI; MICCAI BraTS; 3D DWT; random forest

\section{INTRODUCTION}

A brain tumor is the abnormal growth of tissues inside the brain. Glial cells are the most abundant cell types in the central nervous system. These glial cells surround the neurons and provide insulation and support. A tumor found in these glial cells is called a glioma tumor. The brain is the most common occurrence of these glioma tumors [1]. Three types of glial cells cause brain tumors i.e. oligodendrocytes, astrocytes, and ependymal cells [2]. The glioma tumors produced by these cells are classified into four categories i.e. necrosis, edema, enhancing, and non-enhancing tumors [3].

Brain glioma tumor detection is a complex task that requires skilled analysis to identify the location of the tumor. MR Imaging (MRI) and Computed Tomography (CT) are some of the imaging modalities which are used by radiologists to diagnose the brain tumor. MRI scans are preferred over other radiation based methods because it uses large magnets to locate the cells inside the brain [4]. Better structural image contrast is the goal of all brain imaging procedures. In MRI scans, the tissue relaxation properties
(T1 and T2) contribute to the brightness and contrast of the scanned image [5]. These MRI scans are captured in different sequences i.e. T1, T2, T1c, and flair based on the Repetition Time (TR), Time to Echo (TE), contrast and brightness values. Manual detection of a tumor from an MR image is a long and tedious process. Various image processing techniques are used for automatic detection and classification of the brain tumors [6]. DWT is a method used in image processing to extract feature vectors of an image [7]. These feature vectors are then used to classify the glioma tumor. Supervised and unsupervised classification techniques are used to classify images into tumorous and non-tumorous types. In supervised classification, the input feature vectors are divided into different categories by comparing them with an input training set [8]. The training set is acquired from actual patients diagnosed with glioma tumor.

Once the images are classified, the tumor location can be found through image segmentation, in which Image is divided into various segments for analyses. In medical imaging, different segmentation methods like support vector machines, fuzzy logic, and Markov random fields are used to locate the tumorous parts of the image [9].

The detected glioma tumor, after segmentation, can be categorized into different classes. For this purpose, multilabel classification methods like random forest trees, nearest neighbors, Radial Basis Function (RBF), Naïve Bayes and Multi-Layer Perceptron (MLP) can be used [10]. These classification methods require different features of the tumor like the texture, neighborhood and contextual information. These features can be extracted using various methods like Discrete Cosine Transform (DCT), DWT and Discrete Fourier Transform (DFT) [11].

In this paper, different types of Glioma tumor classification and segmentation is done using block based 3D DWT features. Section II provides the related work in this field and section III discusses the proposed method. Section IV contains the experimental results and the conclusion is reported in the last section.

\section{LITERATURE SURVEY}

In medical image processing, significant work is performed to detect brain tumor in MR images using automated image segmentation. Balafar et al. [9] reviewed different segmentation methods to extract brain tumor from the input MRI scans. However, once the tumor is detected, it 